\title{
Extended signal-correction observer and application to aircraft navigation
}

\author{
Xinhua Wang, Weicheng Wang
}

\begin{abstract}
An extended signal-correction observer (ESCO) is presented for signals correction and estimation, which not only can reject the large measurement error, but also the system uncertainty can be estimated, in spite of the existence of intense stochastic non-Gaussian noise. Multiinput describing function method is proposed to analyze the ESCO robustness in frequency domain. The ESCOs are developed for position/velocity and attitude angle/angular rate integrations, respectively, and they are applied to an aircraft navigation: Based on the ESCO, the position, attitude angle and the uncertainties in the flight dynamics are estimated. Experiments demonstrate the effectiveness of the proposed method.
\end{abstract}

Index Terms-Extended signal-correction observer (ESCO), large measurement error, aircraft.

\section{INTRODUCTION}

$\mathbf{U}$ SUALLY controlling an aircraft needs the information of the position and attitude. Moreover, many aircrafts are underactuated dynamic systems with highly uncertain behaviors: unmodelled dynamics, parametric uncertainties and aerodynamic disturbance are inevitable. These uncertainties render great challenges in flight control.

Global positioning system (GPS) can provide the recognition of position and velocity $[1,2]$. The positioning accuracy by GPS is several meters even more than $10 \mathrm{~m}$, and it is easily contaminated by the circumstances [2]. There are usually two methods of velocity measurement using GPS: 1) Position difference; 2) Doppler shift measurement [3]. The former approach is simplest, but it has a meter per second level of accuracy due to the dependence on position accuracy. In contrast, Doppler shift measurement enables velocity accuracy of a few centimeters per second, even the accuracy approaching $5 \mathrm{~mm} / \mathrm{s}$ is possible [4]. Hence, measuring Doppler shift is a preferred way of velocity measurement. Alternatively, the velocity of device can be estimated by the optical flow sensors (OFS) [5]. Nevertheless, they are sensible to lighting changes. The state observers [6] are popular to estimate velocity from position. However, the above observers are only fit for the systems with relatively accurate position measurements.

Manuscript received August 02, 2018; revised January 02, 2019; accepted March 25, 2019. This work was supported in part by Huatimesee UAV Design Company. (Corresponding author: Xinhua Wang.)

Xinhua Wang is with the Department of Electrical and Electronic Engineering, University of Nottingham, Nottingham, NG7 2RD, U.K. (email: xinhua.wang1@nottingham.ac.uk).

Weicheng Wang is with the Huatimesee UAV Design Company, Beijing, China (e-mail: weiicheng.wang@gmail.com).
Inertial navigation system (INS) can provide position and velocity information, but it suffers from signal drifts over time. Even with the accurate velocity, the drift in position still exist by integration over long time period. Some observer-based INS methods were used [7,8,9]. In [8], a dynamic observer was designed to estimate position, by which the position is required to be in a small bounded range, and it is not fit for large-range navigation. Also, inertial measurement unit (IMU) can provide the attitude information. To calculate the attitude angles of device, the angular rates from the gyroscopes in IMU are onefold integrated, however, the drifts are inevitable. Alternatively, the large-error bounded pitch and roll angles can be determined by the outputs of the accelerometer in IMU, and the large-error bounded yaw angle can be calculated by the outputs of the magnetometer in IMU [10].

Disturbances and uncertainties always exist in aircraft systems. The extended state observers have provided a powerful tool to estimate system disturbances and uncertainties $[11,12,13]$. However, these observers need the relatively accurate position measurements as the observer inputs.

The signals integration (for example, position/acceleration, position/velocity, or attitude angle/angular rate) based on Kalman filter (KF) or Extended Kalman filter (EKF) can restrict the defects of individual systems [14,15,16], and it can improve the accuracy of system outputs. However, for KF or EKF, noise is assumed to be zero-mean Gaussian distributed, and the process noise covariance is uncorrelated to the estimate error. As stochastic non-Gaussian noise exists in signal, the inaccurate noise information may lead to position and attitude drifts. Furthermore, these methods cannot deal with the effect of system uncertainties.

In this paper, injecting the merits of the robustness of finitetime-stable systems [17,18] and high-gain technique [19], an ESCO is presented to reject the large sensor errors in position/attitude, and synchronously to estimate the uncertainty in spite of the existence of stochastic non-Gaussian noise. The multi-input describing function method is proposed to analyze the ESCO robustness in frequency domain. As an application, the ESCO is applied to an experiment on aircraft navigation. In recently years, many researchers investigate the research of aircraft navigation and control [20,21]. Traditionally, aircraft navigation is based on KF [22], and noise is assumed to be be zero-mean Gaussian distributed. In this paper, the following scenario of aircraft navigation is considered: large sensor errors and uncertainties exist in position and attitude; stochastic non-Gaussian noise exists in the velocity and angular rate; the ESCOs are adopted to estimate the position, attitude angle 
and system uncertainties. The performance of the ESCO is compared with the EKF. Finally, a control law based on the ESCOs is designed to stabilize the flight.

\section{Problem description}

The goal of the ESCO design is to reject the large measurement error, and to estimate the system uncertainty.

\section{A. Case of position/velocity integration}

GPS provides the large-error position and accurate velocity of device by Doppler shift measurement. Also, the uncertainties and disturbances exist in position dynamics.

Question 1: How to estimate the accurate position and the system uncertainties in spite of the large-error position measurement and stochastic non-Gaussian noise?

\section{B. Case of attitude angle/angular rate integration}

The gyroscopes in IMU provide the direct measurement of accurate angular rate. The large-error bounded pitch, roll and yaw angles can be determined by the outputs of the accelerometer and magnetometer in IMU, respectively.

Question 2: How to estimate the accurate attitude angles and the attitude uncertainties in spite of the large-error attitude angle measurements and stochastic non-Gaussian noise?

\section{Configuration of ESCO}

The following underactuated system has a minimum number of states and inputs, but it retains the features that must be considered for many systems:

$$
\begin{aligned}
\dot{x}_{1} & =x_{2} \\
\dot{x}_{2} & =h(t)+\sigma(t) \\
y_{o 1} & =x_{1}+d_{1}(t) \\
y_{o 2} & =x_{2}+d_{2}(t)
\end{aligned}
$$

where, $x_{1}$ and $x_{2}$ are the states; $y_{o 1}=x_{1}+d_{1}(t)$ and $y_{o 2}=$ $x_{2}+d_{2}(t)$ are the measurement outputs; $d_{1}(t)$ is the unknown large sensor error, and $\sup _{t \in[0, \infty)}\left|d_{1}(t)\right| \leq L_{1}<\infty ; d_{2}(t)$ is the relatively small sensor error, $\sup _{t \in[0, \infty)}\left|d_{2}(t)\right| \leq L_{2}<$ $\infty$, and $L_{2} \ll L_{1} ; h(t) \in R$ is the known function, and it includes the controller and the other known terms; $\sigma(t) \in R$ is the system uncertainty, and $\sup _{t \in[0, \infty)}|\sigma(t)| \leq L_{3}<\infty$.

1) Extended system of (1)

Assumption 1: Suppose the frequency of uncertainty $\sigma(t)$ in system (1) is far smaller than the system sampling frequency, and its varying

$$
\dot{\sigma}(t)=c_{\sigma}(t)
$$

is bounded, i.e., $c_{\sigma}(t)$ is unknown and bounded, and $\sup _{t \in[0, \infty)}\left|c_{\sigma}(t)\right| \leq L_{a}<\infty$. In fact, the uncertainties in almost all engineering applications are satisfied with this assumption, for instance, crosswind dynamics.

In system (1), uncertainty $\sigma(t)$ is defined as a new state, i.e., $x_{3}=\sigma(t)$. Thus, $\dot{x}_{3}=\dot{\sigma}(t)=c_{\sigma}(t)$ holds. Then, system (1) can be augmented equivalently into

$$
\begin{aligned}
\dot{x}_{1} & =x_{2} \\
\dot{x}_{2} & =x_{3}+h(t) \\
\dot{x}_{3} & =c_{\sigma}(t) \\
y_{o 1} & =x_{1}+d_{1}(t) \\
y_{o 2} & =x_{2}+d_{2}(t)
\end{aligned}
$$

2) ESCO configuration

The configuration of an ESCO for system (3) can be constructed by

$$
\begin{aligned}
& \dot{\hat{x}}_{1}=\widehat{x}_{2} \\
& \dot{\hat{x}}_{2}=\widehat{x}_{3}+h(t) \\
& \dot{\hat{x}}_{3}=f\left(\widehat{x}_{1}-y_{o 1}, \widehat{x}_{2}-y_{o 2}, \widehat{x}_{3}\right)
\end{aligned}
$$

with the conclusions:

$$
\widehat{x}_{1} \rightarrow x_{1}(t), \widehat{x}_{2} \rightarrow x_{2}(t), \widehat{x}_{3} \rightarrow x_{3}
$$

In the ESCO configuration (4), the input signals include the measurements $y_{o 1}$ and $y_{o 2}$. The states $\widehat{x}_{1}, \widehat{x}_{2}$ and $\widehat{x}_{3}$ estimate the real signals $x_{1}, x_{2}$ and uncertainty $x_{3}$ (i.e., $\sigma(t))$, respectively. Importantly, the large sensor error $d_{1}(t)$ in measurement $y_{o 1}$ will be rejected sufficiently, and the drift will be avoided even the non-Gaussian noise exists.

\section{DESIGN OF ESCO}

Considering the large sensor error and stochastic nonGaussian noise, an ESCO is designed to to reject the measurement error and to estimate the system uncertainty, and one theorem is presented as follows.

\section{A. ESCO design}

Theorem 1: Considering the underactuated system (1) (or (3)), for observer

$$
\begin{aligned}
\dot{\hat{x}}_{1}= & \widehat{x}_{2} \\
\dot{\widehat{x}}_{2}= & \widehat{x}_{3}+h(t) \\
\varepsilon^{4} \dot{\hat{x}}_{3}= & -k_{1}\left|\varepsilon\left(\widehat{x}_{1}-y_{o 1}\right)\right|^{\alpha_{1}} \operatorname{sign}\left(\widehat{x}_{1}-y_{o 1}\right) \\
& -k_{2}\left|\widehat{x}_{2}-y_{o 2}\right|^{\alpha_{2}} \operatorname{sign}\left(\widehat{x}_{2}-y_{o 2}\right) \\
& -k_{3}\left|\varepsilon^{3} \widehat{x}_{3}\right|^{\alpha_{3}} \operatorname{sign}\left(\widehat{x}_{3}\right)
\end{aligned}
$$

where, $\varepsilon \in(0,1)$ is the small parameter;

$$
k_{1}>0, k_{3}>0, k_{2}>\varepsilon^{2 \alpha_{2}} k_{1} / k_{3}
$$

and $\alpha_{1}, \alpha_{2}, \alpha_{3}$ satisfy

$$
\alpha_{3} \in(0,1), \alpha_{2}=\frac{\alpha_{3}}{2-\alpha_{3}}, \alpha_{1}=\frac{\alpha_{3}}{3-2 \alpha_{3}}
$$

1) if the sensor errors exist in the measurement signals $y_{01}$ and $y_{o 2}$, i.e., $y_{o 1}=x_{1}+d_{1}(t)$ and $y_{o 2}=x_{2}+d_{2}(t)$, where $x_{1}$ and $x_{2}$ are the states in system (3), $d_{1}(t)$ and $d_{2}(t)$ are the measurement errors, $\sup _{t \in[0, \infty)}\left|d_{1}(t)\right| \leq L_{1}<\infty$, 
$\sup _{t \in[0, \infty)}\left|d_{2}(t)\right| \leq L_{2}<\infty$, and $L_{2} \ll L_{1}$, then there exist $\gamma>\frac{4}{\alpha_{1}}$ and $\Gamma>0$, such that, for $t \geq \varepsilon \Gamma(\Xi(\varepsilon) e(0))$,

$$
\widehat{x}_{i}-x_{i}=O\left(\delta_{d i}^{\gamma}\right), i=1,2,3
$$

where, $O\left(\delta_{d i}^{\gamma}\right)$ means that the error between $\widehat{x}_{i}$ and $x_{i}$ is of order $O\left(\delta_{d i}^{\gamma}\right)[19] ; \delta_{d i} \in(0,1), i=1,2,3 ; \gamma=(1-\beta) / \beta, \beta \in$ $\left(0, \alpha_{1} /\left(\alpha_{1}+4\right)\right) ; \Xi(\varepsilon)=\operatorname{diag}\left\{\varepsilon, \varepsilon^{2}, \varepsilon^{3}\right\}$, and $e_{i}=\widehat{x}_{i}-x_{i}(t)$, $i=1,2,3 ; e=\left[\begin{array}{lll}e_{1} & e_{2} & e_{3}\end{array}\right]^{T}$.

2) Furthermore, if sensor error in signal $y_{o 2}$ is small enough and can be ignored, i.e., $y_{o 2}=x_{2}$, then, even large sensor error exists in signal $y_{o 1}$, for $t \geq \varepsilon \Gamma(\Xi(\varepsilon) e(0))$,

$$
\widehat{x}_{i}-x_{i}=O\left(\varepsilon^{\alpha_{1} \gamma-i}\right), i=1,2,3
$$

where, $O\left(\varepsilon^{\alpha_{1} \gamma-i}\right)$ means that the error between $\widehat{x}_{i}$ and $x_{i}$ is of order $O\left(\varepsilon^{\alpha_{1} \gamma-i}\right)$ [19]. The proof of Theorem 1 is presented in Appendix.

\section{B. Robustness analysis in frequency domain}

In practice, high-frequency noises exist in measurement signals $y_{o 1}$ and $y_{o 2}$. For the ESCO, a multi-input describing function method is proposed to analyze the nonlinear behaviors of the ESCO. The order of nonlinear system is not restricted by this method. The basic idea of describing function method is to replace the actual output of nonlinear elements under the sinusoidal input signal by using the fundamental component in the output signal. Even though it is only an approximation method, the desirable properties it inherits from the frequency response method, and the shortage of other systematic tools for nonlinear system analysis, make it an indispensable tool. Through this method, we can find that the ESCO leads to perform accurate estimation and strong rejection of high-frequency noise.

For system (3), let $\xi_{1}=x_{1}, \xi_{2}=x_{2}, \xi_{3}=x_{3}+h(t)$ and $\dot{h}(t)=\eta(t)$, then $\dot{x}_{3}+\dot{h}(t)=c_{\sigma}(t)+\eta(t)$. Therefore, system (3) can be rewritten as

$$
\dot{\xi}_{1}=\xi_{2} ; \dot{\xi}_{2}=\xi_{3} ; \dot{\xi}_{3}=c_{\sigma}(t)+\eta(t)
$$

Then, for system (11), the ESCO (6) becomes

$$
\begin{aligned}
\dot{\widehat{x}}_{1}= & \widehat{x}_{2} ; \dot{\widehat{x}}_{2}=\widehat{x}_{3} ; \\
\varepsilon^{4} \dot{\widehat{x}}_{3}= & -k_{1}\left|\varepsilon\left(\widehat{x}_{1}-y_{o 1}\right)\right|^{\alpha_{1}} \operatorname{sign}\left(\widehat{x}_{1}-y_{o 1}\right) \\
& -k_{2}\left|\widehat{x}_{2}-y_{o 2}\right|^{\alpha_{2}} \operatorname{sign}\left(\widehat{x}_{2}-y_{o 2}\right) \\
& -k_{3}\left|\varepsilon_{3}^{3} \widehat{x}_{3}\right|^{\alpha_{3}} \operatorname{sign}\left(\widehat{x}_{3}\right)
\end{aligned}
$$

where, $\widehat{x}_{1}, \widehat{x}_{2}$ and $\widehat{x}_{3}$ estimate $\xi_{1}, \xi_{2}$ and $\xi_{3}$, respectively.

The frequency characteristic of ESCO (12) is analysed as follows.

In ESCO (12), for the nonlinear function $|*|^{\alpha_{i}} \operatorname{sign}(*)$, by selecting the input signal as $*=A \sin (\omega t)$, its describing function can be given by:

$$
\begin{aligned}
& N_{i}(A) \\
= & \frac{2}{A \pi} \int_{0}^{\pi}|A \sin (\omega \tau)|^{\alpha_{i}} \operatorname{sign}(A \sin (\omega \tau)) \sin (\omega \tau) d \omega \tau \\
= & \frac{\Omega\left(\alpha_{i}\right)}{A^{1-\alpha_{i}}}
\end{aligned}
$$

where $\Omega\left(\alpha_{i}\right)=\frac{2}{\pi} \int_{0}^{\pi}|\sin (\omega \tau)|^{\alpha_{i}+1} d \omega \tau$. Therefore, the approximation of ESCO (12) through the describing function method is

$$
\begin{aligned}
\dot{\widehat{x}}_{1}= & \widehat{x}_{2} ; \dot{\widehat{x}}_{2}=\widehat{x}_{3} ; \\
\varepsilon^{4} \dot{\widehat{x}}_{3}= & -k_{1} \frac{\Omega\left(\alpha_{1}\right)}{A^{1-\alpha_{1}}} \varepsilon\left(\widehat{x}_{1}-y_{o 1}\right) \\
& -k_{2} \frac{\Omega\left(\alpha_{2}\right)}{A^{1-\alpha_{2}}}\left(\widehat{x}_{2}-y_{o 2}\right)-k_{3} \frac{\Omega\left(\alpha_{3}\right)}{A^{1-\alpha_{3}}} \varepsilon^{3} \widehat{x}_{3}
\end{aligned}
$$

Because $y_{o 1}$ is the integral of $y_{o 2}$, equivalently, $y_{o 2}$ can be taken as the unique input signal. The Laplace transformations of (14) is written as

$$
\begin{aligned}
s \widehat{X}_{1}(s)= & \widehat{X}_{2}(s) ; s \widehat{X}_{2}(s)=\widehat{X}_{3}(s) \\
\varepsilon^{4} s \widehat{X}_{3}(s)= & -k_{1} \frac{\Omega\left(\alpha_{1}\right)}{A^{1-\alpha_{1}}} \varepsilon\left(\widehat{X}_{1}(s)-\frac{1}{s} Y_{o 2}(s)\right) \\
& -k_{2} \frac{\Omega\left(\alpha_{2}\right)}{A^{1-\alpha_{2}}}\left(\widehat{X}_{2}(s)-Y_{o 2}(s)\right) \\
& -k_{3} \frac{\Omega\left(\alpha_{3}\right)}{A^{1-\alpha_{3}}} \varepsilon_{3}^{3} \widehat{X}(s)
\end{aligned}
$$

where $\widehat{X}_{i}(s)$ and $Y_{o 2}(s)$ denote the Laplace transformations of $x_{i}$ and $y_{o 2}$, respectively, and $s$ is the Laplace operator.

From (15), the following transfer functions are determined:

$$
\begin{aligned}
\frac{\widehat{X}_{j}(s)}{Y_{o 2}(s)} & =\frac{k_{2} \frac{\Omega\left(\alpha_{2}\right)}{A^{1-\alpha_{2}}} s^{j-1}+\varepsilon k_{1} \frac{\Omega\left(\alpha_{1}\right)}{A^{1-\alpha_{1}}} s^{j-2}}{\varepsilon^{4} s^{3}+\varepsilon^{3} k_{3} \frac{\Omega\left(\alpha_{3}\right)}{A^{1-\alpha_{3}}} s^{2}+k_{2} \frac{\Omega\left(\alpha_{2}\right)}{A^{1-\alpha_{2}} s+\varepsilon k_{1} \frac{\Omega\left(\alpha_{1}\right)}{A^{1-\alpha} \alpha_{1}}}} \\
j & \in\{1,2,3\}
\end{aligned}
$$

The effects of the ESCO parameters on the robustness are analyzed as follows.

Frequency characteristic with different $\varepsilon$ and $\alpha_{3}$. With different selections of $\alpha_{3}$, we get the values of $\Omega\left(\alpha_{3}\right), \Omega\left(\alpha_{2}\right)$ and $\Omega\left(\alpha_{1}\right)$ shown in Table I.

TABLE I

VALUES OF $\Omega\left(\alpha_{3}\right), \Omega\left(\alpha_{2}\right)$ AND $\Omega\left(\alpha_{1}\right)$

\begin{tabular}{cccc}
\hline \hline$\alpha_{3}$ & $\Omega\left(\alpha_{3}\right)$ & $\Omega\left(\alpha_{2}\right)$ & $\Omega\left(\alpha_{1}\right)$ \\
\hline 0.8 & 1.0410 & 1.0712 & 1.0944 \\
0.5 & 1.1128 & 1.1596 & 1.1852 \\
0.3 & 1.1697 & 1.2093 & 1.2270 \\
\hline \hline
\end{tabular}

For the ESCO, the parameters are selected as follows: $k_{1}=0.02, k_{2}=16, k_{3}=5 ; \alpha_{3}=\alpha=0.8,0.5,0.3 ;$ $\varepsilon=0.6,0.4,0.25$, respectively. The Bode plots of (16) with different selections of $\varepsilon$ and $\alpha_{3}$ are described in Fig.1: Figs.1(a)-(c) present the frequency characteristics of the $\xi_{1}, \xi_{2}$ and $\xi_{3}$ estimations, respectively.

Comparing with ideal operators $1,1 / s$ and $s$, not only the ESCO can obtain their estimations precisely, but also the highfrequency noise is rejected sufficiently. Parameter $\varepsilon$ affects the low-pass frequency bandwidth: Decreasing $\varepsilon$, the low-pass frequency bandwidth becomes larger, and the estimate speed becomes fast; increasing $\varepsilon$, the low-pass frequency bandwidth becomes smaller, and much noise will be rejected. Parameter $\alpha_{3} \in(0,1)$ affects the estimate precision: smaller $\alpha_{3} \in(0,1)$ 


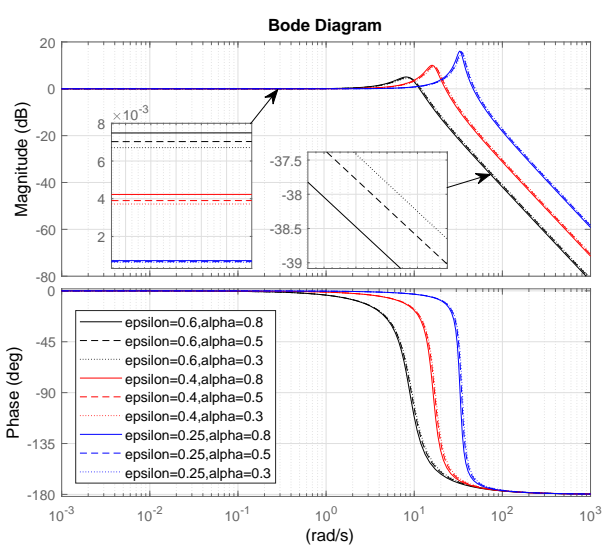

(a)

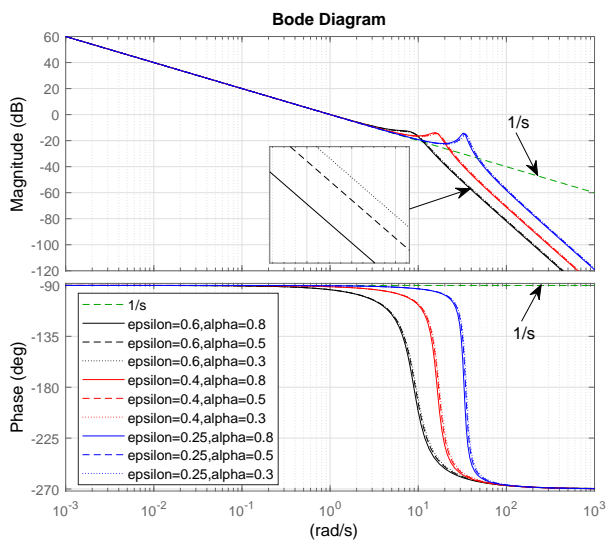

(b)

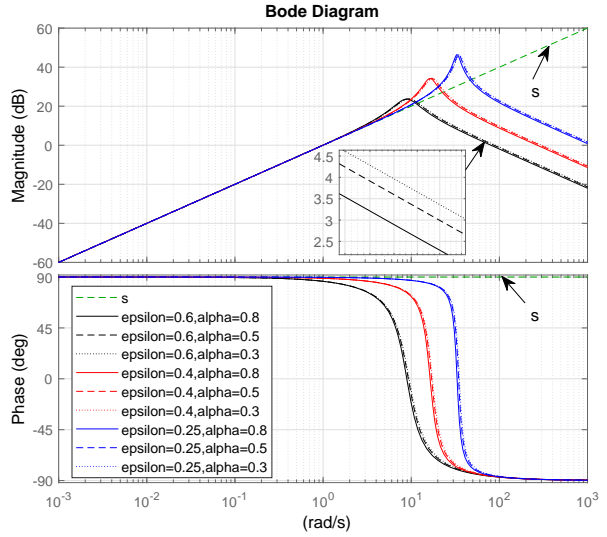

(c)

Fig. 1. Frequency characteristics with changes of $\varepsilon$ and $\alpha$ for ESCO. (a) $\xi_{2}$ estimate. (b) $\xi_{1}$ estimate. (c) $\xi_{3}$ estimate.

can obtain more precise estimations; on the other hand, larger $\alpha_{3} \in(0,1)$ can reduce much noise.

Remark 1 (ESCO analysis):

1) Large sensor error rejection and uncertainty estimation: In ESCO (6), $\widehat{x}_{i}$ estimates, respectively, the state $x_{i}$ of system (3), $i=1,2,3$. In the estimate error (10), due to $\varepsilon \in(0,1)$ and $\alpha_{1} \gamma-i \gg 1$, the estimate error $O\left(\varepsilon^{\alpha_{1} \gamma-i}\right)$ is sufficiently small in spite of the large sensor error $d_{1}(t)$ (where, $\sup _{t \in[0, \infty)}\left|d_{1}(t)\right| \leq L_{1}<\infty$ ) in the measurement output $y_{o 1}=x_{1}+d_{1}(t)$. Therefore, the ESCO performs strong rejection of sensor errors, and the accurate estimations are achieved. Moreover, from the frequency-domain analysis in Fig.1, the ESCO shows the strong rejection of high-frequency noise.

2) No drift: From (9), in spite of the large sensor error and non-Gaussian noise, the estimate errors are always small enough. Therefore, even for unbounded position navigation, no drift exists.

Remark 2 (The rules of ESCO parameters selection):

1) Stability condition: The parameters $\left(k_{1}, k_{2}, k_{3}\right)$ and $\left(\alpha_{1}, \alpha_{2}, \alpha_{3}\right)$ are satisfied with (7) and (8), respectively.

2) Sensor error rejection: When the sensor error $d_{1}(t)$ in $y_{o 1}$ increases, i.e., $L_{1}$ becomes larger, in order to reduce the error effect $k_{1} L_{1}^{\alpha_{1}}$ of $\delta_{0}=\sum_{i=1,3} 2^{1-\alpha_{i}} k_{i} L_{i}^{\alpha_{i}}+L_{a}$ in (35), parameter $k_{1}>0$ should decrease. Meanwhile, $\alpha_{1} \in(0,1)$ should decrease to make $L_{1}^{\alpha_{1}}$ smaller.

3) Filtering: If much noise exists, $\varepsilon \in(0,1)$ should increase, or $\alpha_{3} \in(0,1)$ increases, to make the low-pass frequency bandwidth narrow (See Fig. 1).

\section{Aircraft naVigation based on ESCO}

A quadrotor aircraft navigation is studied. In this scenario, the large-error measurements of position and attitude angle are considered. The forces and torques of aircraft are described in Fig. 2, and its parameters are given in Table II.

\section{A. Quadrotor aircraft dynamics}

Let $\Xi_{g}=\left(E_{x}, E_{y}, E_{z}\right)$ and $\Xi_{b}=\left(E_{x}^{b}, E_{y}^{b}, E_{z}^{b}\right)$ denote the inertial and fuselage frames, respectively; $\psi, \theta$ and $\phi$ are the yaw, pitch and roll angles, respectively. $F_{i}=b \omega_{i}^{2}$ is the thrust force by rotor $i$, and its reactive torque is $Q_{i}=k \omega_{i}^{2}$. The total thrust by the four rotors is given by $F=\sum_{i=1}^{4} F_{i}$. The motion equations of the aircraft in the coordinate $(x, y, z)$ are then

$$
\begin{aligned}
m \ddot{x} & =u_{x}-k_{x} \dot{x}+\Delta_{x} \\
m \ddot{y} & =u_{y}-k_{y} \dot{y}+\Delta_{y} \\
m \ddot{z} & =u_{z}-m g-k_{z} \dot{z}+\Delta_{z} \\
J_{\psi} \ddot{\psi} & =u_{\psi}-k_{\psi} \dot{\psi}+\Delta_{\psi} \\
J_{\theta} \ddot{\theta} & =u_{\theta}-l k_{\theta} \dot{\theta}+\Delta_{\theta} \\
J_{\phi} \ddot{\phi} & =u_{\phi}-l k_{\phi} \dot{\phi}+\Delta_{\phi}
\end{aligned}
$$

where, $k_{x}, k_{y}, k_{z}, k_{\psi}, k_{\theta}$ and $k_{\phi}$ are the unknown drag coefficients; $\left(\Delta_{x}, \Delta_{y}, \Delta_{z}\right)$ and $\left(\Delta_{\psi}, \Delta_{\theta}, \Delta_{\phi}\right)$ are the uncertainties in position and attitude dynamics, respectively; $J=\operatorname{diag}\left\{J_{\psi}, J_{\theta}, J_{\phi}\right\}$ is the matrix of the three-axial moment of inertias; the symbols $c_{\theta}$ and $s_{\theta}$ are used for $\cos \theta$ and $\sin \theta$, respectively, and 


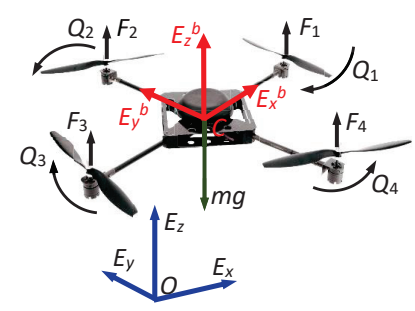

Fig. 2. Forces and torques of quadrotor aircraft.

TABLE II

QuAdROTOR AIRCRAFT PARAMETERS

\begin{tabular}{lll}
\hline \hline Symbol & \multicolumn{1}{c}{ Quantity } & \multicolumn{1}{c}{ Value } \\
\hline$m$ & mass of aircraft & $2.01 \mathrm{~kg}$ \\
$g$ & gravity & $9.81 \mathrm{~m} / \mathrm{s}^{2}$ \\
$l$ & distance between rotor and gravity center & $0.2 \mathrm{~m}$ \\
$J_{\phi}$ & moment of inertia about roll & $1.25 \mathrm{~kg} \cdot \mathrm{m}^{2}$ \\
$J_{\theta}$ & moment of inertia about pitch & $1.25 \mathrm{~kg} \cdot \mathrm{m}^{2}$ \\
$J_{\psi}$ & moment of inertia about yaw & $2.5 \mathrm{~kg} \cdot \mathrm{m}^{2}$ \\
$b$ & rotor force coefficient & $2.923 \times 10^{-3}$ \\
$k$ & Rotor torque coefficient & $5 \times 10^{-4}$ \\
\hline \hline
\end{tabular}

$$
\begin{aligned}
& u_{x}=\left(c_{\psi} s_{\theta} c_{\phi}+s_{\psi} s_{\phi}\right) F, u_{y}=\left(s_{\psi} s_{\theta} c_{\phi}-c_{\psi} s_{\phi}\right) F \\
& u_{z}=c_{\theta} c_{\phi} F, u_{\psi}=\frac{k}{b} \sum_{i=1}^{4}(-1)^{i+1} F_{i} \\
& u_{\theta}=\left(F_{3}-F_{1}\right) l, u_{\phi}=\left(F_{2}-F_{4}\right) l
\end{aligned}
$$

\section{B. Measurement outputs}

GPS provides the position and velocity measurements. IMU can give the attitude angle and angular rate, respectively. The sensor outputs are defined as:

$$
y_{o 1 *}=*+d_{1 *}(t), y_{o 2 *}=\dot{*}+d_{2 *}(t)
$$

where, $*=\{x, y, z, \psi, \theta, \phi\}, \dot{*}=\{\dot{x}, \dot{y}, \dot{z}, \dot{\psi}, \dot{\theta}, \dot{\phi}\} ; d_{1 *}(t)$ and $d_{2 *}(t)$ are the sensor errors, $\sup _{t \in[0, \infty)}\left|d_{1 *}(t)\right| \leq L_{1 *}<\infty$, $\sup _{t \in[0, \infty)}\left|d_{2 *}(t)\right| \leq L_{2 *}<\infty$, and $L_{2 *} \ll L_{1 *}$.

The ESCOs are used to estimate $(x, y, z, \psi, \theta, \phi)$ and the system uncertainties from the large-error measurements.

\section{Controller design}

In this section, the control laws are designed to stabilize the flight. For the reference position and attitude $\left(x_{d}, y_{d}, z_{d}\right)$ and $\left(\psi_{d}, \theta_{d}, \phi_{d}\right)$, the system errors of position (17) and attitude (18) can be written, respectively, by

$$
\ddot{e}_{p}=m^{-1}\left(u_{p}+\Xi_{p}+\delta_{p}\right)
$$

and

$$
\ddot{e}_{a}=J^{-1}\left(u_{a}+\Xi_{a}+\delta_{a}\right)
$$

where, $e_{1}=x-x_{d}, e_{2}=\dot{x}-\dot{x}_{d}, e_{3}=y-y_{d}, e_{4}=\dot{y}-\dot{y}_{d}$, $e_{5}=z-z_{d}, e_{6}=\dot{z}-\dot{z}_{d} ; e_{7}=\psi-\psi_{d}, e_{8}=\dot{\psi}-\dot{\psi}_{d}$, $e_{9}=\theta-\theta_{d}, e_{10}=\dot{\theta}-\dot{\theta}_{d}, e_{11}=\phi-\phi_{d}, e_{12}=\dot{\phi}-\dot{\phi}_{d}$;

$$
\begin{aligned}
& e_{p}=\left[\begin{array}{l}
e_{1} \\
e_{3} \\
e_{5}
\end{array}\right], \delta_{p}=\left[\begin{array}{c}
\Delta_{x}-k_{x} \dot{x} \\
\Delta_{y}-k_{y} \dot{y} \\
\Delta_{z}-k_{z} \dot{z}
\end{array}\right], \\
& u_{p}=\left[\begin{array}{c}
u_{x} \\
u_{y} \\
u_{z}
\end{array}\right], \Xi_{p}=\left[\begin{array}{c}
-m \ddot{x}_{d} \\
-m \ddot{y}_{d} \\
-m \ddot{z}_{d}-m g
\end{array}\right]
\end{aligned}
$$

and

$$
\begin{gathered}
e_{a}=\left[\begin{array}{c}
e_{7} \\
e_{9} \\
e_{11}
\end{array}\right], \Xi_{a}=\left[\begin{array}{c}
-J_{\psi} \ddot{\psi}_{d} \\
-J_{\theta} \ddot{\theta}_{d} \\
-J_{\phi} \ddot{\phi}_{d}
\end{array}\right], \\
u_{a}=\left[\begin{array}{c}
u_{\psi} \\
u_{\theta} \\
u_{\phi}
\end{array}\right], \delta_{a}=\left[\begin{array}{c}
\Delta_{\psi}-k_{\psi} \dot{\psi} \\
\Delta_{\theta}-l k_{\theta} \dot{\theta} \\
\Delta_{\phi}-l k_{\phi} \dot{\phi}
\end{array}\right]
\end{gathered}
$$

1) Position dynamics controller: For position dynamics (17), to track reference position $\left(x_{d}, y_{d}, z_{d}\right)$, the controller

$$
u_{p}=-\Xi_{p}-\widehat{\delta}_{p}-m\left(k_{p 1} \widehat{e}_{p}+k_{p 2} \widehat{\dot{e}}_{p}\right)
$$

can be selected to make position error (21) converge to the origin asymptotically, i.e., $e_{p} \rightarrow 0$ and $\dot{e}_{p} \rightarrow 0$ as $t \rightarrow \infty$, where $\widehat{e}_{1}=\widehat{x}-x_{d}, \widehat{e}_{2}=\widehat{\dot{x}}-\dot{x}_{d}, \widehat{e}_{3}=\widehat{y}-y_{d}, \widehat{e}_{4}=\widehat{\dot{y}}-\dot{y}_{d}$, $\widehat{e}_{5}=\widehat{z}-z_{d}, \widehat{e}_{6}=\stackrel{\dot{z}}{-} \dot{z}_{d}$ and $\widehat{\delta}_{p}$ are estimated by the ESCOs; $k_{p 1}, k_{p 2}>0$, and

$$
\widehat{e}_{p}=\left[\begin{array}{lll}
\widehat{e}_{1} & \widehat{e}_{3} & \widehat{e}_{5}
\end{array}\right]^{T}, \widehat{\dot{e}}_{p}=\left[\begin{array}{lll}
\widehat{e}_{2} & \widehat{e}_{4} & \widehat{e}_{6}
\end{array}\right]^{T}
$$

2) Attitude dynamics controller: For attitude dynamics (18), to track reference attitude $\left(\psi_{d}, \theta_{d}, \phi_{d}\right)$, the controller

$$
u_{a}=-\Xi_{a}-\widehat{\delta}_{a}-J\left(k_{a 1} \widehat{e}_{a}+k_{a 2} \widehat{\dot{e}}_{a}\right)
$$

can be selected to make attitude error (22) converge to the origin asymptotically, i.e., $e_{a} \rightarrow 0$ and $\dot{e}_{a} \rightarrow 0$ as $t \rightarrow \infty$, where, $\widehat{e}_{7}=\widehat{\psi}-\psi_{d}, \widehat{e}_{8}=\widehat{\dot{\psi}}-\dot{\psi}_{d}, \widehat{e}_{9}=\widehat{\theta}-\theta_{d}, \widehat{e}_{10}=\widehat{\dot{\theta}}-\dot{\theta}_{d}$, $\widehat{e}_{11}=\widehat{\phi}-\phi_{d}, \widehat{e}_{12}=\widehat{\dot{\phi}}-\dot{\phi}_{d}$ and $\widehat{\delta}_{a}$ are estimated by the ESCOs; $k_{a 1}, k_{a 2}>0$, and

$$
\widehat{e}_{a}=\left[\begin{array}{lll}
\widehat{e}_{7} & \widehat{e}_{9} & \widehat{e}_{11}
\end{array}\right]^{T}, \widehat{\dot{e}}_{a}=\left[\begin{array}{lll}
\widehat{e}_{8} & \widehat{e}_{10} & \widehat{e}_{12}
\end{array}\right]^{T}
$$

\section{EXPERIMENT ON AIRCRAFT NAVIGATION}

In this section, an experiment is presented to illustrate the proposed scheme. The platform of aircraft navigation and control is shown in Fig. 3, and the implementation of the navigation strategy based on the ESCO is done in the platform setup. The control system hardware is described in Fig.4, whose components are: Gumstix and an Arduino Mega 2560 (sampling frequency: $16 \mathrm{MHz}$ ) are taken as the driven boards; A XsensMTI AHRS (sampling frequency: $10 \mathrm{kHz}$ ) is used to provide the angular rate, acceleration and earth's magnetic field; The control update time is $5 \mathrm{~ms}$. 


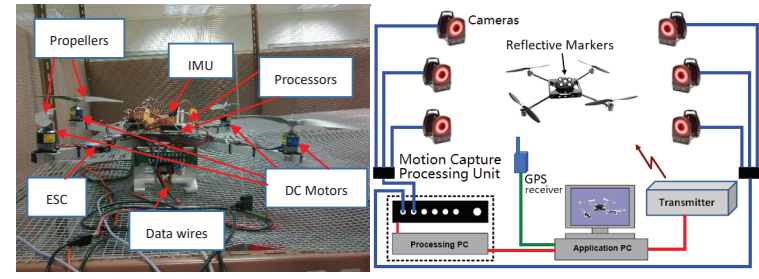

Fig. 3. Platform of aircraft system.

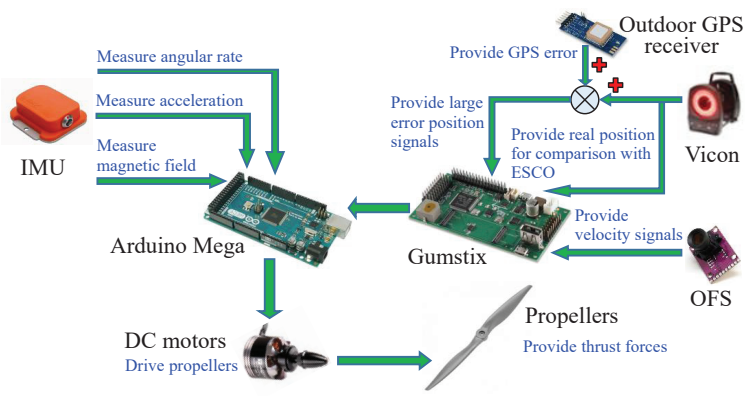

Fig. 4. Control system hardware.

Real position: In order to obtain the real position for comparison with the estimate by the ESCO, the output of the Vicon system (i.e., indoor positioning system) with an accuracy of sub-millimetre is taken as the real position.

Large-error position measurement: As GPS receiver cannot receive position signal in lab, we use the output of the Vicon contaminated by the prior GPS error signal as the large-error position measurement (See Fig. 4). The GPS error signal was collected through an outdoor test: the position signal of a static GPS receiver at the origin was recorded with time. To make the GPS error more obvious, the recorded signal magnified 4 times. Then, we got the GPS error signal.

Velocity measurement: A XZN OFS board (up to $6400 \mathrm{fps}$ update rate, $30 \times 30$ pixel resolution) is adopted to measure the velocity instead of the GPS Doppler shift measurement. The room light can be tuned to obtain the accurate OFS measurement of velocity.

Reference trajectory: The reference trajectory consists of take-off and a circle with the radius $5 \mathrm{~m}$, velocity $1 \mathrm{~m} / \mathrm{s}$ and altitude $3 \mathrm{~m}$, which is shown in Fig. 5 .

The ESCO provides the estimations of the position and uncertainties, which are replaced into the controller. Controllers (25) and (27) are used to drive the aircraft to track the reference trajectory. The parameters of ESCOs: $\alpha_{i, 3}=0.5$, $k_{i, 1}=0.05, k_{i, 2}=16, k_{i, 3}=3,1 / \varepsilon_{i}=1.5, i=1,2,3$. The controller gains: $k_{p 1}=2.5, k_{p 2}=4, k_{a 1}=2.5, k_{a 2}=4$. The ESCO performance is studied through the behavior of estimated position and uncertainties, and compared with the estimate results of the EKF proposed in [16].

Fig. 5(a) shows the flight trajectories, including the measured, real and desired trajectories, the estimated trajectories by the ESCO and EKF. Meanwhile, the position estimate comparisons in the three directions are shown in Fig. 5(b): The position measurement errors are about $20 \mathrm{~m}$. The estimated errors by the ESCO are less than $0.05 \mathrm{~m}$, while the up-

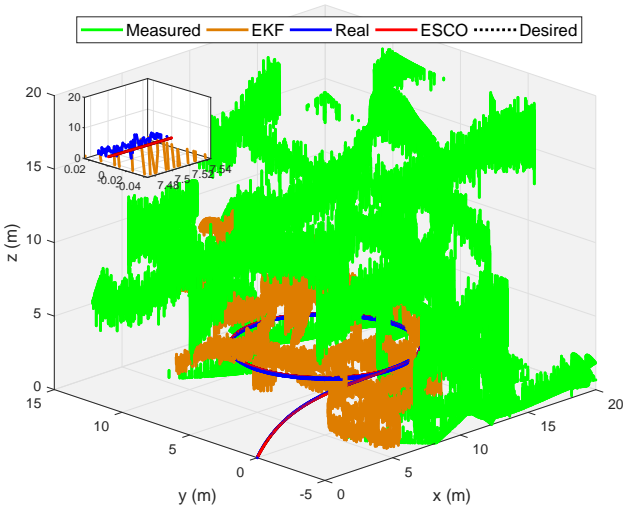

(a)

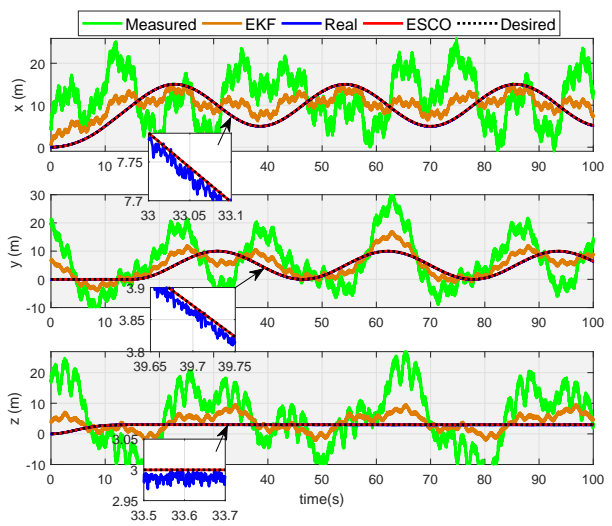

(b)

Fig. 5. Aircraft navigation based on ESCO. (a) Navigation trajectories. (b) Position estimate.

boundness of the estimate errors by the EKF is at least $5 \mathrm{~m}$. Thus, the large measurement errors are rejected by the ESCO, and the effect of stochastic noises is reduced sufficiently. Even in the long-time flight test (1000s), no drift happened. Reversely, the larger estimate errors exist in the outputs of the EKF comparing to the ESCO. Furthermore, it cannot deal with the adverse effects of system uncertainties and stochastic non-Gaussian noise.

Uncertainties estimation in simulation: Under our present condition, we cannot obtain the real uncertainties in experiment for comparison. Therefore, a simulation is presented to verify the ESCO performance of uncertainties estimation.

Uncertain parameters in aircraft: $k_{x}=k_{y}=k_{z}=$ $0.01 \mathrm{Ns} / \mathrm{m}, k_{\psi}=k_{\theta}=k_{\phi}=0.012 \mathrm{Ns} / \mathrm{rad}$. The disturbances are selected as $\Delta_{x}=\sin (0.8 t), \Delta_{y}=0.8 \sin (t)$, $\Delta_{z}=0.6 \sin (0.5 t)$. The measurement errors are the GPS error signals in the experiment part. Therefore, the real uncertainty vector $\delta_{p}$ can be determined from (23). The parameters of the ESCO and controllers are selected as the same values in the above experiment. Fig. 6 illustrates the uncertainties estimation, where the performance by the ESCO showed the accurate estimations. 

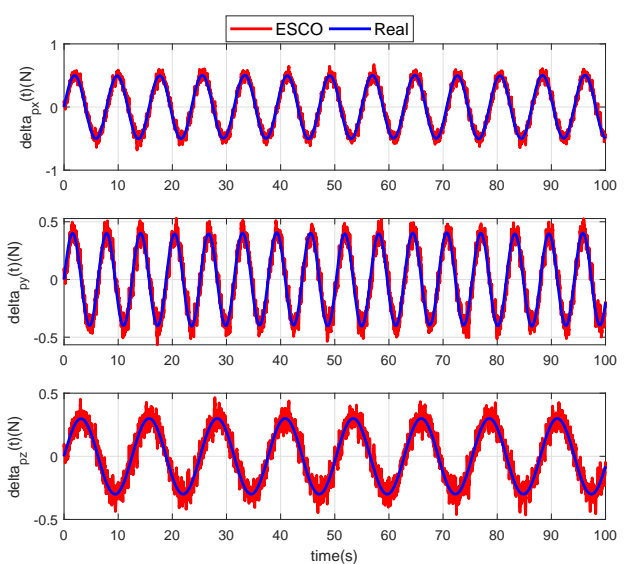

Fig. 6. Uncertainties estimation.

\section{CONCLUSIONS}

An ESCO has been developed to reject the large sensor error and to estimate the system uncertainty in spite of the existence of stochastic non-Gaussian noise. The proposed ESCO demonstrated by an aircraft navigation experiment: it succeeded in rejecting the large sensor errors in position, and the system uncertainties were estimated accurately. The merits of the presented ESCO include its rejections of large sensor errors and stochastic non-Gaussian noise, uncertainty estimation and no drift.

\section{APPENDIX}

Proof of Theorem 1: The system error of ESCO (6) and system (3) can be described by:

$$
\begin{aligned}
\dot{e}_{1}= & e_{2} ; \dot{e}_{2}=e_{3} ; \\
\varepsilon^{4} \dot{e}_{3}= & -k_{1}\left|\varepsilon\left(e_{1}-d_{1}(t)\right)\right|^{\alpha_{1}} \operatorname{sign}\left(e_{1}-d_{1}(t)\right) \\
& -k_{2}\left|e_{2}-d_{2}(t)\right|^{\alpha_{2}} \operatorname{sign}\left(e_{2}-d_{2}(t)\right) \\
& -k_{3}\left|\varepsilon^{3}\left(e_{3}+\sigma(t)\right)\right|^{\alpha_{3}} \operatorname{sign}\left(e_{3}+\sigma(t)\right) \\
& -\varepsilon^{4} c_{\sigma}(t)
\end{aligned}
$$

and Eq. (29) can be rewritten as

$$
\begin{aligned}
\frac{d \varepsilon e_{1}}{d t / \varepsilon}= & \varepsilon^{2} e_{2} ; \frac{d \varepsilon^{2} e_{2}}{d t / \varepsilon}=\varepsilon^{3} e_{3} ; \\
\frac{d \varepsilon^{3} e_{3}}{d t / \varepsilon}= & -k_{1}\left|\varepsilon e_{1}-\varepsilon d_{1}(t)\right|^{\alpha_{1}} \operatorname{sign}\left(e_{1}-d_{1}(t)\right) \\
& -\frac{k_{2}}{\varepsilon^{2 \alpha_{2}}}\left|\varepsilon^{2} e_{2}-\varepsilon^{2} d_{2}(t)\right|^{\alpha_{2}} \operatorname{sign}\left(e_{2}-d_{2}(t)\right) \\
& -k_{3}\left|\varepsilon^{3} e_{3}+\varepsilon^{3} \sigma(t)\right|^{\alpha_{3}} \operatorname{sign}\left(e_{3}+\sigma(t)\right) \\
& -\varepsilon^{4} c_{\sigma}(t)
\end{aligned}
$$

By choosing the following coordinate transform

$$
\begin{aligned}
\tau & =t / \varepsilon, z_{i}(\tau)=\varepsilon^{i} e_{i}, z=\left[\begin{array}{lll}
z_{1} & z_{2} & z_{3}
\end{array}\right]^{T} ; \\
\bar{d}_{i}(\tau) & =\varepsilon^{i} d_{i}(t), i=1,2 ; \bar{d}_{3}(\tau)=\varepsilon^{3} \sigma(t) ; \\
\bar{d}_{4}(\tau) & =\varepsilon^{4} c_{\sigma}(t)
\end{aligned}
$$

we get $z=\Xi(\varepsilon) e$, where, $\Xi(\varepsilon)=\operatorname{diag}\left\{\varepsilon, \varepsilon^{2}, \varepsilon^{3}\right\}$ and $e=$ $\left[\begin{array}{lll}e_{1} & e_{2} & e_{3}\end{array}\right]^{T}$. Then, (30) becomes

$$
\begin{aligned}
\frac{d z_{1}}{d \tau}= & z_{2} ; \frac{d z_{2}}{d \tau}=z_{3} ; \\
\frac{d z}{d \tau}= & -k_{1}\left|z_{1}-\bar{d}_{1}(\tau)\right|^{\alpha_{1}} \operatorname{sign}\left(z_{1}-\bar{d}_{1}(\tau)\right) \\
& -\frac{k_{2}}{\varepsilon^{2 \alpha_{2}}}\left|z_{2}-\bar{d}_{2}(\tau)\right|^{\alpha_{2}} \operatorname{sign}\left(z_{2}-\bar{d}_{2}(\tau)\right) \\
& -k_{3}\left|z_{3}+\bar{d}_{3}(\tau)\right|^{\alpha_{3}} \operatorname{sign}\left(z_{3}+\bar{d}_{3}(\tau)\right) \\
& -\bar{d}_{4}(\tau)
\end{aligned}
$$

Define

$$
\begin{aligned}
& g(\tau, z(\tau)) \\
= & -k_{1}\left\{\left|z_{1}-\bar{d}_{1}(\tau)\right|^{\alpha_{1}} \operatorname{sign}\left(z_{1}-\bar{d}_{1}(\tau)\right)\right. \\
& \left.-\left|z_{1}\right|^{\alpha_{1}} \operatorname{sign}\left(z_{1}\right)\right\} \\
& -\frac{k_{2}}{\varepsilon^{2 \alpha_{2}}}\left\{\left|z_{2}-\bar{d}_{2}(\tau)\right|^{\alpha_{2}} \operatorname{sign}\left(z_{2}-\bar{d}_{2}(\tau)\right)\right. \\
& \left.-\left|z_{2}\right|^{\alpha_{2}} \operatorname{sign}\left(z_{2}\right)\right\}-\bar{d}_{4}(\tau) \\
& -k_{3}\left\{\left|z_{3}+\bar{d}_{3}(\tau)\right|^{\alpha_{3}} \operatorname{sign}\left(z_{3}+\bar{d}_{3}(\tau)\right)\right. \\
& \left.-\left|z_{3}\right|^{\alpha_{3}} \operatorname{sign}\left(z_{3}\right)\right\}
\end{aligned}
$$

then, (32) can be rewritten as

$$
\begin{aligned}
\frac{d z_{1}}{d \tau}= & z_{2} ; \frac{d z_{2}}{d \tau}=z_{3} \\
\frac{d z_{3}}{d \tau}= & -\sum_{i=1}^{2} k_{i}\left|z_{i}\right|^{\alpha_{i}} \operatorname{sign}\left(z_{i}\right) \\
& -\frac{k_{3}}{\varepsilon^{3 \alpha_{3}}}\left|\left(z_{3}\right)\right|^{\alpha_{3}} \operatorname{sign}\left(z_{3}\right)+g(\tau, z(\tau))
\end{aligned}
$$

Since the contraction mapping rule $\left|x^{\rho_{i}}-\bar{x}^{\rho_{i}}\right| \leq$ $2^{1-\rho_{i}}|x-\bar{x}|^{\rho_{i}}, \rho_{i} \in(0,1]$, we obtain

$$
\begin{aligned}
\delta & =\sup _{(\tau, z) \in R^{4}}|g(\tau, z(\tau))| \\
& \leq \sum_{i=1,3} 2^{1-\alpha_{i}} k_{i} L_{i}^{\alpha_{i}} \varepsilon^{i \alpha_{i}}+\varepsilon^{4} L_{a}+2^{1-\alpha_{2}} k_{2} L_{2}^{\alpha_{2}} \\
& \leq \varepsilon^{\rho} \delta_{0}+2^{1-\alpha_{2}} k_{2} L_{2}^{\alpha_{2}}
\end{aligned}
$$

where $\delta_{0}=\sum_{i=1,3} 2^{1-\alpha_{i}} k_{i} L_{i}^{\alpha_{i}}+L_{a}$, and $\rho=$ $\min _{i \in\{1,3\}}\left\{\min \left\{4, i \alpha_{i}\right\}\right\}=\alpha_{1}$.

From Proposition 8.1 in [17], Theorem 5.2 in [18] and (35), for (32), there exist the bounded constants $\mu>0$ and $\Gamma(z(0))>0$, such that, for $\tau \geq \Gamma(z(0))$,

$$
\|z(\tau)\| \leq \mu \delta^{\gamma} \leq \mu\left(\varepsilon^{\alpha_{1}} \delta_{0}+2^{1-\alpha_{2}} k_{2} L_{2}^{\alpha_{2}}\right)^{\gamma}
$$

Therefore, from (31), we get

$$
\left\|\varepsilon e_{1} \quad \varepsilon^{2} e_{2} \quad \varepsilon^{3} e_{3} \quad\right\| \leq \mu\left(\varepsilon^{\alpha_{1}} \delta_{0}+2^{1-\alpha_{2}} k_{2} L_{2}^{\alpha_{2}}\right)^{\gamma}
$$

for $t \geq \varepsilon \Gamma(\Xi(\varepsilon) e(0))$. Thus, the following relation holds: 


$$
e_{i}=O\left(\delta_{d i}^{\gamma}\right), i=1,2,3, \forall t \in[\varepsilon \Gamma(\Xi(\varepsilon) e(0)), \infty)
$$

where, $\delta_{d i}=\varepsilon^{\alpha_{1}-\frac{i}{\gamma}}+\frac{2^{1-\alpha_{2}} k_{2} L_{2}^{\alpha_{2}}}{\delta_{0}} \varepsilon^{-\frac{i}{\gamma}}, i=1,2,3$. If $\varepsilon \in$ $(0,1)$ and $L_{2}<\left(\frac{1-\varepsilon^{\alpha_{1}}}{2^{1-\alpha_{2}} k_{2}} \delta_{0}\right)^{\frac{1}{\alpha_{2}}}$, then

$$
0<\varepsilon^{\alpha_{1}}+\frac{2^{1-\alpha_{2}}}{\delta_{0}} k_{2} L_{2}^{\alpha_{2}}<1
$$

Furthermore, from Theorems 4.3 and 5.2 in [18], $\gamma$ is arbitrarily large. Hence, the requirement that $\gamma$ lies on

$$
\gamma>\max \left\{\frac{4 \log \varepsilon}{\log \left(\varepsilon^{\alpha_{1}}+\frac{2^{1-\alpha_{2}}}{\delta_{0}} k_{2} L_{2}^{\alpha_{2}}\right)}, 1\right\}
$$

is not restrictive. Therefore, we obtain

$$
\gamma \log \left(\varepsilon^{\alpha_{1}}+\frac{2^{1-\alpha_{2}}}{\delta_{0}} k_{2} L_{2}^{\alpha_{2}}\right)<4 \log \varepsilon
$$

i.e.,

$$
\varepsilon^{\alpha_{1}}+\frac{2^{1-\alpha_{2}}}{\delta_{0}} k_{2} L_{2}^{\alpha_{2}}<\varepsilon^{\frac{4}{\gamma}}
$$

From (40), $\gamma>4$ holds. Therefore, from $\varepsilon \in(0,1)$, we can get $\varepsilon^{\frac{4}{\gamma}}<\varepsilon^{\frac{i}{\gamma}}, i=1,2,3$. Then

$$
\delta_{d i}=\varepsilon^{\alpha_{1}-\frac{i}{\gamma}}+\frac{2^{1-\alpha_{2}}}{\delta_{0}} k_{2} L_{2}^{\alpha_{2}} \varepsilon^{-\frac{i}{\gamma}}<1
$$

where $i=1,2,3$. The choice of $\gamma$ leads to $\gamma>4$ in (38), and it implies that, for $\delta_{d i} \in(0,1)$, the estimate error in (38) is of higher order than the perturbation. Consequently, the ESCO leads to perform rejection of persistent disturbances.

Furthermore, if sensor error is ignored in signal $y_{o 2}$, i.e, $y_{o 2}=x_{2}$ or $L_{2}=0$, then (38) can be written as

$$
e_{i}=O\left(\varepsilon^{\alpha_{1} \gamma-i}\right), i=1,2,3, \forall t \in[\varepsilon \Gamma(\Xi(\varepsilon) e(0)), \infty)
$$

From Theorems 4.3 and 5.2 in [18], $\gamma$ can be chosen to be arbitrarily large, and

$$
\gamma>\max \left\{\frac{4}{\alpha_{1}}, 1\right\}=\frac{4}{\alpha_{1}}
$$

is not restrictive. Accordingly, we can get $\alpha_{1} \gamma-i>1$ for $i=1,2,3$. It implies that, for $\varepsilon \in(0,1)$, the estimate error in (44) is of higher order than the perturbation.

For $\varepsilon \in(0,1)$, according to the Routh-Hurwitz Stability Criterion, $s^{3}+k_{3} s^{2}+\frac{k_{2}}{\varepsilon^{2 \alpha_{2}}} s+k_{1}$ is Hurwitz if $k_{1}>0, k_{3}>0$, $k_{2}>\varepsilon^{2 \alpha_{2}} k_{1} / k_{3}$. This concludes the proof.

\section{REFERENCES}

[1] D. Odijk, N. Nadarajah, S. Zaminpardaz, P.J. G. Teunissen. GPS, Galileo, QZSS and IRNSS differential ISBs: estimation and application, GPS Solutions, vol. 21, no. 2, 439-450, Apr. 2017.

[2] M.F. Abdel-Hafez. Detection of bias in GPS satellites' measurements: A probability ratio test formulation, IEEE Trans. Control Syst. Technol., Vol. 22, No. 3, 1166-1173, 2014.
[3] S. Ricci, L. Bassi, P. Tortoli. Real-time vector velocity assessment through multigate doppler and plane waves, IEEE Trans. Ultrason., Ferroelect., Freq. Control, vol. 61, no. 2, pp. 314 324, Feb. 2014.

[4] L. Serrano, D. Kim, R.B. Langley, K. Itani, M. Ueno. A GPS Velocity Sensor: How Accurate Can It Be? - A First Look, ION NTM 2004, 26-28 Jan. 2004, San Diego, CA.

[5] L.R.G. Carrillo, I. Fantoni, and E. Rondon. Three-dimensional position and velocity regulation of a quad-rotorcraft using optical flow, IEEE Trans. Aerosp. Electron. Syst., vol. 51, no. 1, 358-371, 2015.

[6] A. Levant. High-order sliding modes, differentiation and outputfeedback control, Int. J. of Control, vol. 76, no. 9/10, 924-941, 2003.

[7] J.M. Hansen , T.A. Johansen, N. Sokolova, T.I. Fossen. Nonlinear Observer for Tightly Coupled Integrated Inertial Navigation Aided by RTK-GNSS Measurements, IEEE Trans. Control Syst. Technol., DOI: 10.1109/TCST.2017.2785840

[8] X. Wang, B. Shirinzadeh, and M.H. Ang,Jr. Nonlinear doubleintegral observer and application to quadrotor aircraft, IEEE Trans. Ind. Electron., vol. 62, no. 2, 1189-1200, 2015.

[9] R.H. Rogne, T.H. Bryne, T.I. Fossen, T.A. Johansen. Redundant MEMS-based inertial navigation using nonlinear Observers, $J$. Dyn. Sys., Meas., Control, vol. 140, no. 7, 071001 1-7, Jul. 2018.

[10] S.A. Ludwig, A,R. Jiménez. Optimization of gyroscope and accelerometer/magnetometer portion of basic attitude and heading reference system, 2018 IEEE International Symposium on Inertial Sensors and Systems (INERTIAL), Moltrasio, Italy, 2629 Mar. 2018

[11] X. Wang, C. Shi, and S. Wang. Extended state observer-based motion synchronisation control for hybrid actuation system of large civil aircraft, Int. J. Syst. Sci., Vol. 48, no. 10, 2212-2222, 2017.

[12] Z. Pu, R. Yuan, J. Yi, X. Tan. A class of adaptive extended state observers for nonlinear disturbed systems, IEEE Trans. Ind. Electron., vol. 62, no. 9, 5858-5869, Sept. 2015.

[13] J. Liu, S. Vazquez, L. Wu, A. Marquez, H. Gao, L.G. Franquelo. Extended state observer-based sliding-mode control for threephase power converters, IEEE Trans. Ind. Electron., vol. 64, no. 1 , pp. 22 - 31, Jan. 2017.

[14] Y. Kim, J. An, J. Lee. Robust navigational system for a transporter using GPS/INS fusion, IEEE Trans. Ind. Electron., vol. 65, no. 4, 3346-3354, Apr. 2018.

[15] F. Auger, M. Hilairet, J.M. Guerrero, E. Monmasson. Industrial applications of the Kalman filter: A Review, IEEE Trans. Ind. Electron., vol. 60, no. 12, 5458-5471, Dec. 2013.

[16] L. Idkhajine, E. Monmasson, A. Maalouf. Fully FPGA-based sensorless control for synchronous AC drive Using an Extended Kalman filter, IEEE Trans. Ind. Electron., vol. 59, no. 10, 39083918, Oct. 2012.

[17] S.P. Bhat and D.S. Bernstein. Geometric homogeneity with applications to finite-time stability, Math. Control, Signals, Syst., vol. 17, no. 2, 101-127, Jun. 2005.

[18] S.P. Bhat and D.S. Bernstein. Finite-time stability of continuous autonomous systems, Siam J. Control Optim., vol. 38, no. 3, 751-766, Mar. 2000.

[19] H. Kahlil. Nonlinear Systems, 3rd ed. Englewood Cliffs, NJ, USA: Prentice-Hall, 2002, pp. 381-449.

[20] C. Shi, X. Wang, S. Wang, JunWang, and M.M. Tomovic. Adaptive decoupling synchronous control of dissimilar redundant actuation system for large civil aircraft, Aerosp. Sci. Technol., Vol. 47, 114-124, 2015.

[21] K. Sebesta and N. Boizot. A real-time adaptive high-gain EKF, applied to a quadcopter inertial navigation system, IEEE Trans. Ind. Electron., vol. 61, no. 1, pp. 495-503, Jan. 2014.

[22] J.L. Crassidis. Introduction to the Special Issue on the Kalman Filter and Its Aerospace Applications, J. Guid. Control Dyn., vol. 40, no. 9, pp. 2137-2137, 2017. 